\title{
Numerical Modelization of the Oil Film Pressure for a Hydrodynamic Tilting-Pad Thrust Bearing
}

\author{
Le Anh Dung, Tran Thi Thanh Hai * \\ Hanoi University of Science and Technology, No.1 Dai Co Viet str., Hai Ba Trung dist., Hanoi, Vietnam \\ Received: June 17, 2020; Accepted: November 12, 2020
}

\begin{abstract}
This study analyses the hydrodynamic characteristic of the tilting pad thrust bearing. Research content is simultaneously solving the Reynolds equation, force equilibrium equation, and momentum equilibrium equations. Reynolds equation is solved by utilizing the finite element method with Galerkin weighted residual, thereby determines the pressure at each discrete node of the film. Force and momentums are integrated from pressure nodes by Gaussian integral. Finally, force and momentum equilibrium equations are solved using Newton-Raphson iterative to achieve film thickness and inclination angles of the pad at the equilibrium position. The results yielded the film thickness, the pressure distribution on the whole pad and different sections of the bearing respected to the radial direction. The high-pressure zone is located at the low film thickness zone and near the pivot location.
\end{abstract}

Keywords: Hydrodynamic tilting-pad thrust bearing, equilibrium position, finite element method.

\section{Introduction}

Tilting pad thrust bearings are used in rotary machineries, allow for the thrust load operation at the average of rotation and support a heavy load. Hydrodynamic thrust bearing based on hydrodynamic lubrication.

In 2012, D. V. Srikanth et. al. [1] studied a large tilting pad thrust bearing angular stiffness. In 2014, Najar and Harmain [2] performed a numerical study on pressure profiles in the hydrodynamic thrust bearing. Annan Guo et.al [3] experiment static and dynamic characteristics of tilting pad thrust bearing in the same year. In 2018, Mostefa K. et al. [4] analyzed the effect of dimple geometries on textured tilting pad thrust bearing using a finite difference method.

In Vietnam, studies about hydrodynamic tilting pad bearing are few. Most recently, Hai T.T.T et al [5] compared a numerical calculation of a hydrodynamic fixed pad thrust bearing with experiment results. Besides Dung Le Anh et al. [6] perform a numerical modelization of oil film pressure in hydrodynamic journal bearing under a steady load.

This research analyzes the pressure and oil film thickness of a pivot tilting pad thrust bearing at hydrodynamic lubrication.

\section{Thrust bearing and the equations}

\subsection{Tilting pad thrust bearing}

*Corresponding author: Tel.: (+84) 978263926

Email: hai.tranthithanh@hust.edu.vn
Fig. 1 shows the diagram of a pad in pivot tilting pad thrust bearing. Here, the pad is placed onto a pivot that is eccentric from the middle of the pad, to form the oil wedge as hydrodynamic theory. The pressure in the wedge generates the force onto the pad surface making the pad to tilt at an angle respected to the $r$-axis and the $\theta$-axis. $r$ and $\theta$ are two directions of polar coordinates. The inner and outer radius of the pad are $r_{1}$ and $r_{2}, \theta_{p a d}$ is the pad angle, $\theta_{p}$ and $r_{p}$ are the position of the pivot, $h_{p}$ is the film thickness at pivot location, $\alpha_{r}$ and $\alpha_{\theta}$ are the inclinations of the pad along the radial and circumferential direction, $\omega$ is the angular velocity of the collar.

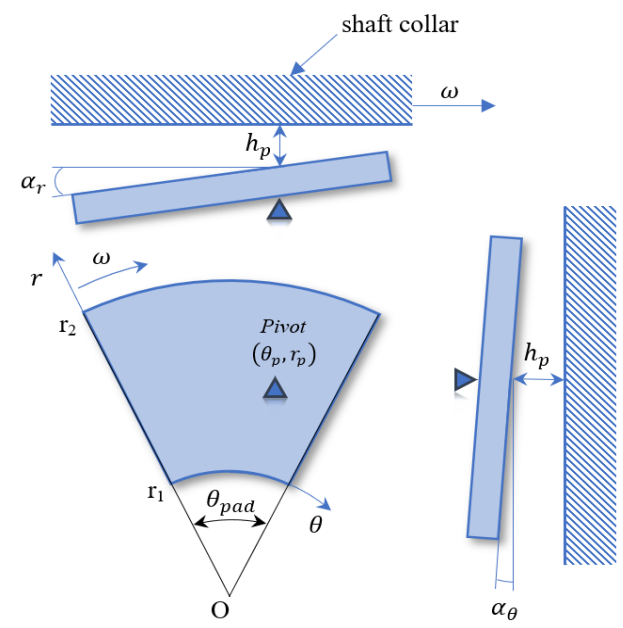

Fig. 1. Diagram of a pivot tilting pad thrust bearing. 


\subsection{The equations}

\subsubsection{The Reynolds equation:}

Obtained from the Naviers-Stockes and continuity equations for an incompressible, isoviscous, steady-state and laminar flow fluid, the Reynolds equation is written in polar coordinates [7]:

$$
\frac{\partial}{\partial r}\left[r h^{3} \frac{\partial p}{\partial r}\right]+\frac{1}{r} \frac{\partial}{\partial \theta}\left[h^{3} \frac{\partial p}{\partial \theta}\right]=6 \mu \omega r \frac{\partial h}{\partial \theta}
$$

where $p$ is oil film pressure, $r$ is radial direction, $\theta$ is circumferential direction, $h$ is oil film thickness, $\mu$ is dynamic viscosity, $\omega$ is angular velocity of the shaft.

The boundary condition chosen for the Reynolds equation is:

$$
\begin{aligned}
& p(\theta=0, r)=0 ; p\left(\theta=\theta_{\text {pad }}, r\right)=0 \\
& p\left(\theta, r=r_{1}\right)=0 ; p\left(\theta, r=r_{2}\right)=0
\end{aligned}
$$

\subsubsection{Oil film thickness equation:}

$$
\begin{aligned}
& h=h_{p}+\left[r_{p}-r \cos \left(\theta-\theta_{p}\right)\right] \sin \left(\alpha_{\theta}\right)+ \\
& +r \sin \left(\theta-\theta_{p}\right) \sin \left(\alpha_{r}\right)
\end{aligned}
$$

\subsubsection{Force and momentum equilibrium equations:}

The pressure distribution is integrated over the pad area to give the resulting force and the moments in two perpendicular directions around the pivot point.

$$
\begin{aligned}
& F_{z}\left(h_{p}, \alpha_{\theta}, \alpha_{r}\right)=\iint_{S} p r d \theta d r=W \\
& M_{x}\left(h_{p}, \alpha_{\theta}, \alpha_{r}\right)=\iint_{S} p r^{2} \sin \left(\theta-\theta_{p}\right) d \theta d r=0 \\
& M_{y}\left(h_{p}, \alpha_{\theta}, \alpha_{r}\right)=\iint_{S} p\left(r \cdot \cos \left(\theta-\theta_{p}\right)-r_{p}\right) r d \theta d r=0
\end{aligned}
$$

\section{Algorithm}

\subsection{Solving Reynolds equation}

The pad domain is discreted into a 4-node finite element mesh as Fig. 2. Here coordinates in polar form is change to the reference coordinates $(\xi, \eta)$. Using finite element method, integrate equation (1) over the domain $\mathrm{S}$ :

$$
\iint_{S} W_{i}\left(\frac{\partial}{\partial r}\left[r h^{3} \frac{\partial p}{\partial r}\right]+\frac{1}{r} \frac{\partial}{\partial \theta}\left[h^{3} \frac{\partial p}{\partial \theta}\right]-6 \mu \omega r \frac{\partial h}{\partial \theta}\right) d S=0
$$

where $W_{i}$ is weight functions.

Integrate by part equation (4), we have:

$$
\begin{aligned}
& \iint_{S}-r h^{3} \frac{\partial W_{i}}{\partial r} \frac{\partial p}{\partial r}-\frac{1}{r} h^{3} \frac{\partial W_{i}}{\partial \theta} \frac{\partial p}{\partial \theta}-W_{i} \cdot 6 \mu \omega r \frac{\partial h}{\partial \theta} d S+ \\
& +\oint W_{i} r h^{3} \frac{\partial p}{\partial r} n_{r} d \tau+\oint_{b} \frac{1}{r} W_{i} \cdot h^{3} \frac{\partial p}{\partial \theta} n_{\theta} d \tau=0
\end{aligned}
$$

$p$ can be expressed as: $p=\sum_{i=1}^{n} N_{i} p_{i}=N .\{p\}$

where $n$ is the total number of mesh points, $N$ is the global polynomials function vector.

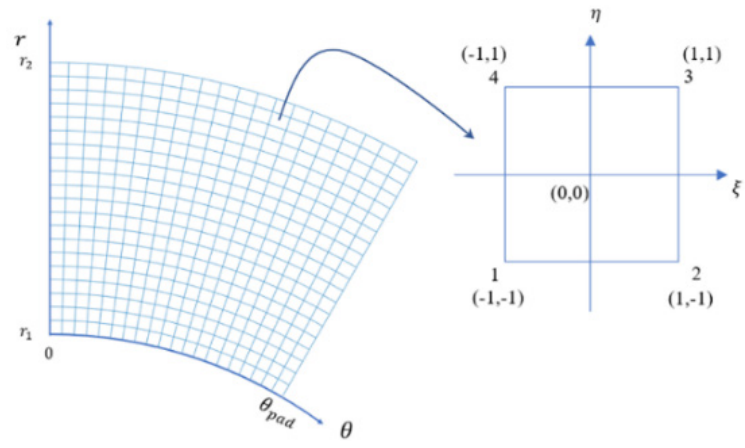

Fig. 2. Diagram of a tilting pad thrust bearing

With Galerkin weight residual, the weighting function $W_{i}$ is chosen equal to the shape function $N_{i}$. Combined with boundary condition, we have:

$\oint W_{i} r h^{3} \frac{\partial p}{\partial r} n_{r} d \tau=0$ and $\oint_{b} \frac{1}{r} W_{i} \cdot h^{3} \frac{\partial p}{\partial \theta} n_{\theta} d \tau=0$

Equation (5) becomes:

$$
\begin{aligned}
& \iint_{S} r h^{3} \frac{\partial\left\{N_{i}\right\}}{\partial r} \frac{\partial\left\{N_{j}\right\}}{\partial r}+\frac{1}{r} h^{3} \frac{\partial\left\{N_{i}\right\}}{\partial \theta} \frac{\partial\left\{N_{j}\right\}}{\partial \theta} d S\{p\} \\
& =\iint_{S}-\left\{N_{i}\right\} \cdot 6 \mu \omega r \frac{\partial h}{\partial \theta} d S
\end{aligned}
$$

Rewrite the left side of equation (4) as a matrix form:

$$
\iint_{S}\left[\begin{array}{cc}
\frac{\partial N_{i}}{\partial \theta} & \frac{\partial N_{i}}{\partial r} \\
\vdots & \vdots \\
\frac{\partial N_{n}}{\partial \theta} & \frac{\partial N_{n}}{\partial r}
\end{array}\right]\left[\begin{array}{cc}
\frac{h^{3}}{r} & 0 \\
0 & r h^{3}
\end{array}\right] \cdot\left[\begin{array}{ccc}
\frac{\partial N_{i}}{\partial \theta} & \cdots & \frac{\partial N_{n}}{\partial \theta} \\
\frac{\partial N_{i}}{\partial r} & \cdots & \frac{\partial N_{n}}{\partial r}
\end{array}\right]\left\{p_{i}\right\} r d \theta d r
$$

The conversion from polar coordinates to reference coordinate system is featured by Jacobi matrix $J$ : 
$J=\left[\begin{array}{ll}\frac{\partial \theta}{\partial \xi} & \frac{\partial r}{\partial \xi} \\ \frac{\partial \theta}{\partial \eta} & \frac{\partial r}{\partial \eta}\end{array}\right]=\left[\begin{array}{cc}\sum_{i=1}^{n} \frac{\partial N_{j}}{\partial \xi} \theta_{i} & \sum_{i=1}^{n} \frac{\partial N_{j}}{\partial \xi} r_{i} \\ \sum_{i=1}^{n} \frac{\partial N_{j}}{\partial \eta} \theta_{i} & \sum_{i=1}^{n} \frac{\partial N_{j}}{\partial \eta} r_{i}\end{array}\right]$

Thus (7) becomes:

$\iint_{S}\left[\begin{array}{cc}\frac{\partial N_{i}}{\partial \theta} & \frac{\partial N_{i}}{\partial r} \\ \vdots & \vdots \\ \frac{\partial N_{n}}{\partial \theta} & \frac{\partial N_{n}}{\partial r}\end{array}\right] \times J^{-1} \times\left[\begin{array}{cc}\frac{h^{3}}{r} & 0 \\ 0 & r h^{3}\end{array}\right] \times$

$\times J^{-1} \times\left[\begin{array}{ccc}\frac{\partial N_{i}}{\partial \theta} & \cdots & \frac{\partial N_{n}}{\partial \theta} \\ \frac{\partial N_{i}}{\partial r} & \cdots & \frac{\partial N_{n}}{\partial r}\end{array}\right] \times\left\{p_{i}\right\} r \times$

$\times \operatorname{det}(J) \times d \xi d \eta=[K] \times\left\{p_{i}\right\}$

The right side of the equation (6) becomes:

$$
\begin{aligned}
\{F\} & =\iint_{S}-\left\{N_{i}\right\} \cdot 6 \mu \omega r \frac{\partial h}{\partial \theta} r d \theta d r \\
& =-\iint_{S}\left\{N_{i}\right\} \cdot 6 \mu \omega r \frac{\partial h}{\partial \theta} r \cdot \operatorname{det}(J) \cdot d \xi d \eta
\end{aligned}
$$

Thus, equation (6) is rewritten as:

$$
[K] .\left\{p_{i}\right\}=\{F\}
$$

Solve the above equation with boundary condition, we can get pressure value at all nodes.

\subsection{Solve force and momentum equation}

Let:

$$
\begin{aligned}
S & =\iint_{S} r d \theta d r \\
R & =\iint_{S} r^{2} \sin \left(\theta-\theta_{p}\right) d \theta d r \\
T & =\iint_{S}\left(r \cdot \cos \left(\theta-\theta_{p}\right)-r_{p}\right) r d \theta d r
\end{aligned}
$$

We get:. $F_{z}\left(h_{p}, \alpha_{\theta}, \alpha_{r}\right)=S^{t}\left\{p_{i}\right\}$

$$
M_{x}\left(h_{p}, \alpha_{\theta}, \alpha_{r}\right)=R^{t}\left\{p_{i}\right\}
$$$$
M_{y}\left(h_{p}, \alpha_{\theta}, \alpha_{r}\right)=T^{t}\left\{p_{i}\right\}
$$

The Jacobian matrix related to the equilibrium position is:

$$
D=-\left[\begin{array}{lll}
\frac{\partial F_{z}}{\partial h_{p}} & \frac{\partial F_{z}}{\partial \alpha_{\theta}} & \frac{\partial F_{z}}{\partial \alpha_{r}} \\
\frac{\partial M_{x}}{\partial h_{p}} & \frac{\partial M_{x}}{\partial \alpha_{\theta}} & \frac{\partial M_{x}}{\partial \alpha_{r}} \\
\frac{\partial M_{y}}{\partial h_{p}} & \frac{\partial M_{y}}{\partial \alpha_{\theta}} & \frac{\partial M_{y}}{\partial \alpha_{r}}
\end{array}\right]
$$

Substitute (13) to (14), we have:

$$
\begin{aligned}
D & =-\left[\begin{array}{lll}
S^{t} \cdot p_{h_{p}} & S^{t} \cdot p_{\alpha_{\theta}} & S^{t} \cdot p_{\alpha_{r}} \\
R^{t} \cdot p_{h_{p}} & R^{t} \cdot p_{\alpha_{\theta}} & R^{t} \cdot p_{\alpha_{r}} \\
T^{t} \cdot p_{h_{p}} & T^{t} \cdot p_{\alpha_{\theta}} & T^{t} \cdot p_{\alpha_{r}}
\end{array}\right] \\
& =-\left\{\begin{array}{l}
S^{t} \\
R^{t} \\
T^{t}
\end{array}\right\}\left[\begin{array}{lll}
p_{h_{p}} & p_{\alpha_{\theta}} & p_{\alpha_{r}}
\end{array}\right]
\end{aligned}
$$

where $p_{h_{p}}=\frac{\partial p}{\partial h_{p}}, p_{\alpha_{\theta}}=\frac{\partial p}{\partial \alpha_{\theta}}, p_{\alpha_{r}}=\frac{\partial p}{\partial \alpha_{r}}$

$p_{h_{p}}, p_{\alpha_{\theta}}, p_{\alpha_{r}}$ are calculated as follows:

Derive equation (11) respected to $h_{p}, \alpha_{\theta}, \alpha_{r}$ :

$\frac{\partial[K]}{\partial k}\left\{p_{i}\right\}+\frac{\partial\left\{p_{i}\right\}}{\partial k}[K]=\frac{\partial\{F\}}{\partial k}$

with $k=h_{p}, \alpha_{\theta}, \alpha_{r}$.

Thus, $\frac{\partial\left\{p_{i}\right\}}{\partial k}=\frac{1}{[K]}\left(\frac{\partial\{F\}}{\partial k}-\frac{\partial[K]}{\partial k}\left\{p_{i}\right\}\right)$

where:

$$
\begin{aligned}
\frac{\partial[K]}{\partial k}= & \iint_{S} 3 h^{2} \frac{\partial h}{\partial k} \times \\
& \times\left(r \frac{\partial\left\{N_{i}\right\}}{\partial r} \frac{\partial\left\{N_{j}\right\}}{\partial r}+\frac{1}{r} \frac{\partial\left\{N_{i}\right\}}{\partial \theta} \frac{\partial\left\{N_{j}\right\}}{\partial \theta}\right) r d \theta d r
\end{aligned}
$$

$\frac{\partial\{F\}}{\partial k}=\iint_{S}-\left\{N_{i}\right\} .6 \mu \omega r \frac{\partial}{\partial k}\left(\frac{\partial h}{\partial \theta}\right) r d \theta d r$

In order to solve the nonlinear equation, NewtonRaphson method is commonly used due to its rapid convergence and highly accurate approximation. Let $u=\left[h_{p}, \alpha_{\theta}, \alpha_{r}\right]^{t}$ be the present step of the equilibrium position, $u^{\text {new }}$ be the new guess value for the new step. Thus, the iterative process is given by: 


$$
u_{\text {new }}=u-D \backslash\left[F_{z}-W, M_{x}-0, M_{y}-0\right]^{t}
$$

This iteration process ends when the following error bound condition err is satisfied:

$$
\frac{u_{n e w}{ }^{k+1}-u^{k}}{\left\|u^{k+1}\right\|} \leq \operatorname{err} \& \frac{F_{z}-W}{F_{z}} \leq \operatorname{err} \& M_{x} \leq e r r
$$

$\& M_{y} \leq e r r$ with $e r r=10^{-5}$.

Within the algorithm described above, programming diagram is shown in Fig.3. A set of initial values are used to calculate the pressure, film thickness and inclinations. Then in each iteration, new values are updated until the solution converges.

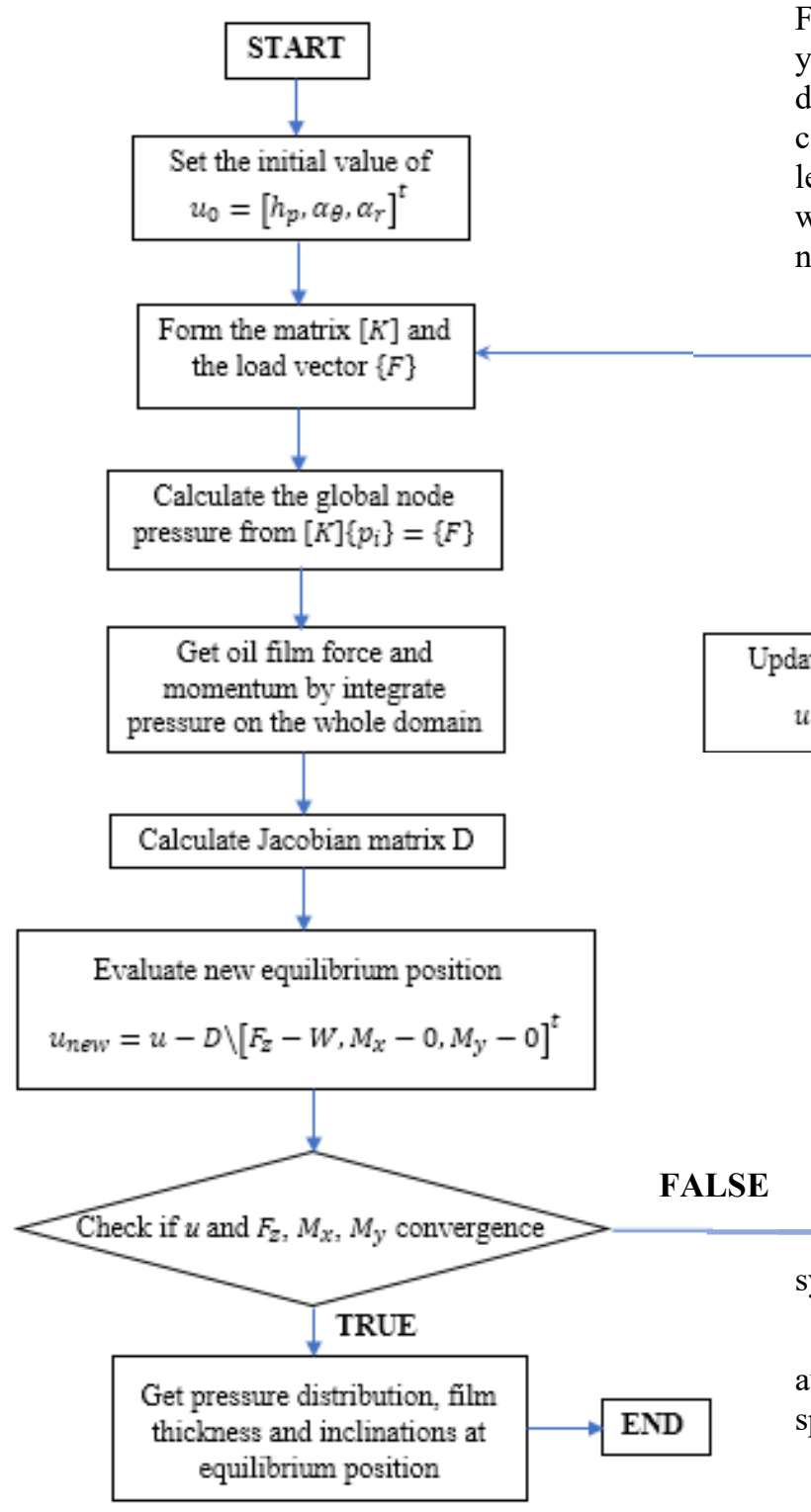
symmetry of the model.

In order to check the validation of the program, author compared with the study of Kouider [4] with specific parameters showed in Table 3.

Fig. 3. Programming Algorithm 


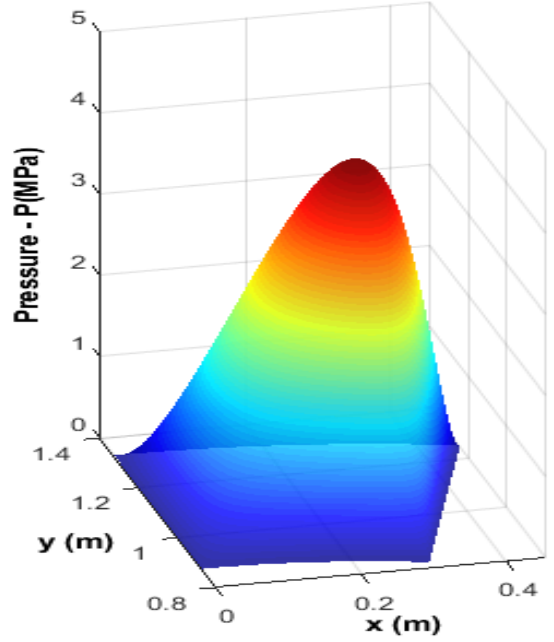

(a)

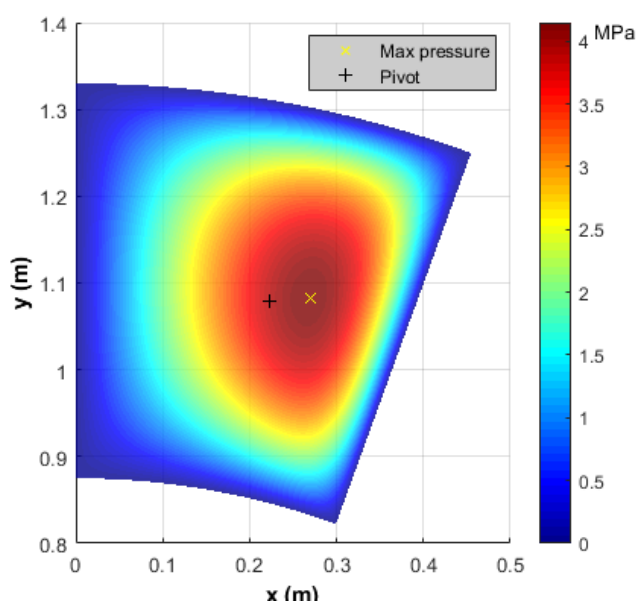

(b)

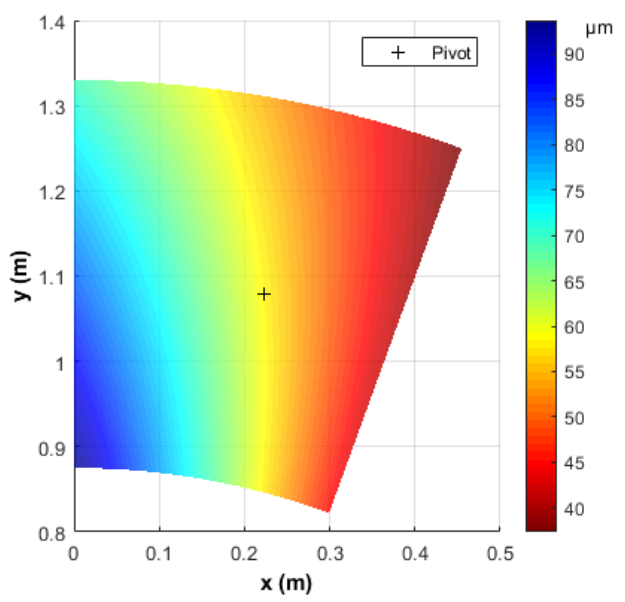

(c)

Fig. 4. (a,b) Pressure distribution of a pad. (c) Film thickness of a pad
Table 1. Bearing parameters

\begin{tabular}{|l|c|}
\hline \multicolumn{1}{|c|}{ Pad Parameters } & Value \\
\hline Rotational speed, $N$ (rpm) & 157 \\
\hline Inner radius/Outer radius, $\frac{r_{1}}{r_{2}}(\mathrm{~m})$ & $0.875 / 1.330$ \\
\hline Dynamic viscosity, $\mu$ (Pa.s) & 0.00565 \\
\hline Number of pads & 12 \\
\hline Pad angle, $\theta_{p a d}(\mathrm{deg})$ & 20 \\
\hline $\begin{array}{l}\text { Pivot circumferential position, } \theta_{p} \\
\text { (deg) }\end{array}$ & 11.652 \\
\hline Pivot radius, $r_{p}(\mathrm{~m})$ & 1.1025 \\
\hline Applied load on each pad $(\mathrm{N})$ & 321667 \\
\hline
\end{tabular}

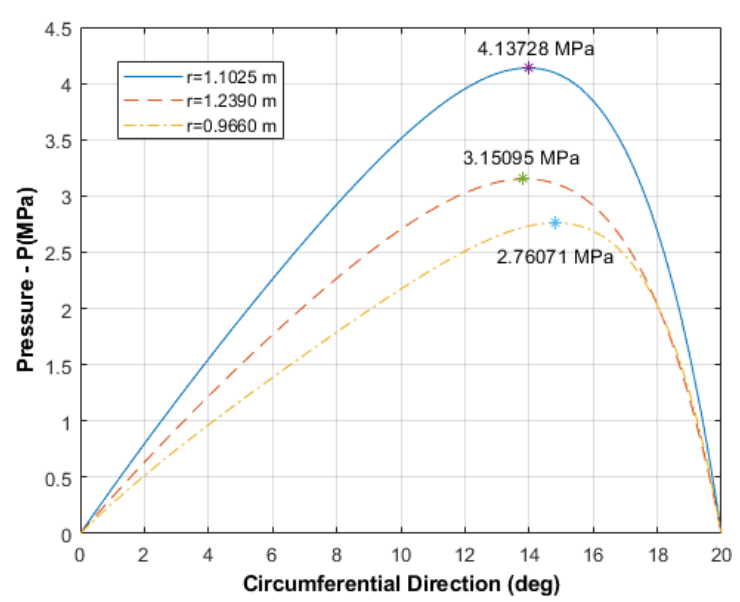

Fig. 5. Pressure distribution in different sections.

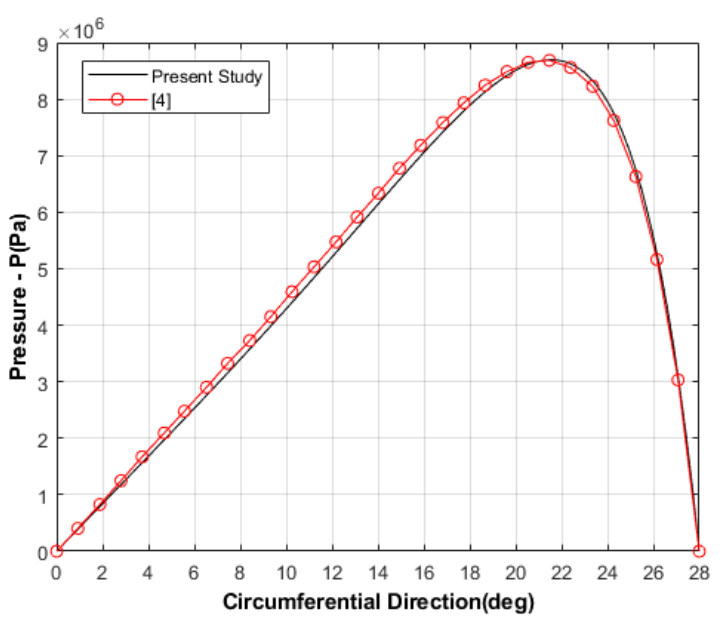

Fig. 6. Compare pressure distribution with [4] 
Table 2. Iteration process within a mesh of $100 \times 100$

\begin{tabular}{|c|l|l|l|c|c|c|c|}
\hline Iteration & $\begin{array}{l}h_{p} \\
(\mu \mathrm{m})\end{array}$ & $\begin{array}{l}\alpha_{r} \\
\left(10^{-5} . \mathrm{rad}\right)\end{array}$ & $\begin{array}{c}\alpha_{\theta} \\
\left(10^{-5} . \mathrm{rad}\right)\end{array}$ & $\begin{array}{c}\text { Max pressure } \\
(\mathrm{MPa})\end{array}$ & $\begin{array}{c}\mathrm{Fz} \\
\left(10^{5} \mathrm{~N}\right)\end{array}$ & $\begin{array}{c}\mathrm{Mx} \\
(\mathrm{Nm})\end{array}$ & $\begin{array}{c}\text { My } \\
(\mathrm{Nm})\end{array}$ \\
\hline 0 & 50.000000 & 10.000000 & 0.000000 & 6.474877 & 4.938925 & 1468.991 & 7251.081 \\
\hline 1 & 56.966530 & 10.976675 & -1.779205 & 4.649763 & 3.595186 & 534.561 & 2265.785 \\
\hline 2 & 58.543082 & 10.842759 & -3.014021 & 4.189871 & 3.247799 & 92.999 & 264.700 \\
\hline 3 & 58.507289 & 10.728756 & -3.184184 & 4.148570 & 3.216814 & 1.612 & 2.445 \\
\hline 4 & 58.503206 & 10.726141 & -3.185541 & 4.148326 & 3.216669 & $6.958 \times 10^{-5}$ & $-1.852 \times 10^{-5}$ \\
\hline 5 & 58.503206 & 10.726140 & -3.185541 & 4.148326 & 3.216670 & $2.182 \times 10^{-11}$ & $-1.107 \times 10^{-10}$ \\
\hline
\end{tabular}

Table 3. Parameters Application for bearing in [4]

\begin{tabular}{|l|c|}
\hline Pad Parameters & Value \\
\hline Rotational speed, $N$ (rpm) & 3000 \\
\hline $\begin{array}{l}\text { Inner radius / Outer radius, } \stackrel{r_{1}}{ } r_{2} \\
(\mathrm{~m})\end{array}$ & $187.5 / 322.5$ \\
\hline Dynamic viscosity, $\mu$ (Pa.s) & 0.0252 \\
\hline Number of pads & 12 \\
\hline Pad angle, $\theta_{p a d}(\mathrm{deg})$ & 28 \\
\hline $\begin{array}{l}\text { Pivot circumferential position, } \theta_{p} \\
(\text { deg})\end{array}$ & 17.38 \\
\hline Pivot radius, $r_{p}(\mathrm{~m})$ & 255.00 \\
\hline Applied load on each pad (N) & 59592 \\
\hline
\end{tabular}

Fig. 6 shows the pressure comparison between the present study with the results in Kouider's calculation [4] in the middle section. While used the finite difference method, there still have slight differences in value. The maximum value in reference is $8.673 \mathrm{MPa}$ while in the present study, this value is $8.708 \mathrm{MPa}$. Overall, both results are in good agreement.

\section{Conclusion}

This research numerically simulates the pressure distribution and the film thickness of tilting pad thrust bearing. The high-pressure zone is located at the lower film thickness zone and near the pivot location. The maximum pressure is not positioned in the middle section of the bearing due to the non-symmetric of the model. The pressure at the different radial sections of the bearing is diverse in value because of the nonsymmetry of the model.
The results of this study are the foundation for future researches taking into account thermal and deformation problems.

\section{References}

[1] D. V. Srikanth, K. K. Chaturvedi, and A. C. K. Reddy, Determination of a large tilting pad thrust bearing angular stiffness, Tribology International, vol. 47, pp. 69-76, 2012

[2] Aa Farooq Ahmad Najar and G. A. Harmain Numerical Investigation of Pressure Profile in Hydrodynamic Lubrication Thrust Bearing, International Scholarly Research Notices (2014) ID157615.

[3] Annan Guo, Xiaojing W., Jian J, Diann Y Hua, Zikai H. Experimental test of static and dynamic characteristics of tilting-pad thrust bearings, Advances in Mechanical Engineering 2015, Vol. 7(7) 1-8

[4] Mostefa Kouider, Souchet D., Zebbar D., Youcefi A. Effects of the dimple geometry on the isothermal performance of a hydrodynamic textured tiltingpad thrust bearing, Journal of Heat and Technology, Vol. 36, No. 2, 2018, pp. 463-472

[5] Hai T.T.T, Thuan L. T. Modeling and Experimental Investigation of Oil Film Pressure Distribution for Hydrodynamic Thrust Bearing, Journal of Science and Technology Technical Universities No.121 (2017) 065-070

[6] Dung L.A, Hai T.T.T, Thuan L.T, Numerical Modelization for Equilibrium Position of a Static Loaded Hydrodynamic Bearing, Journal of Science and Technology Technical Universities No.141 (2020).

[7] Dominique Bonneau, Dominique Souchet, Aurelian Fatu. Hydrodynamic bearing, University of Poitiers, France.2014. 